\title{
No association between variations in extracranial venous anatomy and clinical outcomes in multiple sclerosis patients over 5 years
}

Sirin Gandhi', Karen Marr', Marcello Mancini², Maria Grazia Caprio², Dejan Jakimovski', Avinash Chandra', Jesper Hagemeier ${ }^{1}$, David Hojnacki ${ }^{3}$, Channa Kolb ${ }^{3}$, Bianca Weinstock-Guttman ${ }^{3}$ and Robert Zivadinov $v^{1,4^{*}}$ (D)

\begin{abstract}
Background: No longitudinal, long-term, follow-up studies have explored the association between presence and severity of variations in extracranial venous anatomy, and clinical outcomes in patients with multiple sclerosis (MS).

Objective: This prospective 5-year follow-up study assessed the relationship of variations in extracranial venous anatomy, indicative of chronic cerebrospinal venous insufficiency (CCSVI) on Doppler sonography, according to the International Society for Neurovascular Disease (ISNVD) proposed consensus criteria, with clinical outcomes and disease progression in MS patients.

Methods: 90 MS patients (52 relapsing-remitting, RRMS and 38 secondary-progressive, SPMS) and 38 age- and sexmatched Hls were prospectively followed for 5.5 years. Extracranial and transcranial Doppler-based venous hemodynamic assessment was conducted at baseline and follow-up to determine the extent of variations in extracranial venous anatomy. Change in Expanded Disability Status Scale ( $\triangle E D S S)$, development of disability progression (DP) and annualized relapse rate (ARR) were assessed.
\end{abstract}

Results: No significant differences were observed in MS patients, based on their presence of variations in extracranial venous anatomy at baseline or at the follow-up, in $\triangle E D S S$, development of DP or ARR. While more MS patients had ISNVD CCSVI criteria fulfilled at baseline compared to HIs (58\% vs. 37\%, $p=0.03$ ), no differences were found at the 5-year follow-up (61\% vs. 56\%, $p=0.486)$.

Discussion: This is the longest follow-up study assessing the longitudinal relationship between the presence of variations in extracranial venous anatomy and clinical outcomes in MS patients. Conclusion: The presence of variations in extracranial venous anatomy does not influence clinical outcomes over the 5 -year follow-up in MS patients.

Keywords: Multiple sclerosis, Extracranial, Variations in extracranial venous anatomy, CCSVI, Relapse rate, Disability progression, Longitudinal

\footnotetext{
* Correspondence: rzivadinov@bnac.net

${ }^{1}$ Buffalo Neuroimaging Analysis Center, Department of Neurology, Jacobs

School of Medicine and Biomedical Sciences, University at Buffalo, State

University of New York, Buffalo, NY, USA

${ }^{4}$ Center for Biomedical Imaging at Clinical Translational Science Institute,

University at Buffalo, State University of New York, Buffalo, NY, USA

Full list of author information is available at the end of the article
}

(c) The Author(s). 2019 Open Access This article is distributed under the terms of the Creative Commons Attribution 4.0 International License (http://creativecommons.org/licenses/by/4.0/), which permits unrestricted use, distribution, and

reproduction in any medium, provided you give appropriate credit to the original author(s) and the source, provide a link to the Creative Commons license, and indicate if changes were made. The Creative Commons Public Domain Dedication waiver (http://creativecommons.org/publicdomain/zero/1.0/) applies to the data made available in this article, unless otherwise stated. 


\section{Background}

Multiple sclerosis (MS) is as an autoimmune disease affecting the central nervous system (CNS) that is characterized by demyelination and neurodegeneration from the earliest stages of its clinical presentation [1].

Compared with the arterial system, the development of the extracranial venous anatomy is subject to many variations. In the past, these variations were acknowledged as non-pathological findings Although the possible role of variations in extracranial venous anatomy in the development of the MS was first questioned more than one and a half centuries ago [2], and ever since, sparse cumulative evidence investigating the vascular etiology of MS pathogenesis has been documented over the years [3]. However, it was only recently that a causal association between variations in extracranial venous anatomy suggestive of chronic cerebrospinal venous insufficiency (CCSVI) and MS has been implied [4]. CCSVI is characterized by the variations in of main extracranial cerebrospinal venous outflow routes (obstructions of the internal jugular veins, IJV and/or azygos vein) that interfere with normal venous drainage, as evidenced by Doppler sonography (DS) [4, 5], although other non-invasive [6] and invasive [4, 7, 8] imaging methods were also used to detect these variations in extracranial venous anatomy. These variations in extracranial venous anatomy can be extra- or intra-luminal, and are may be a result of congenital truncular venous malformations, which represent an embryologically defective vein where developmental arrest has occurred during the vascular trunk formation period in the 'later stage' of the embryonic development [6].

Although the condition was originally described in MS patients, the results from independent controlled studies demonstrated that there is highly variable prevalence of this condition in patients with other CNS disorders and in healthy individuals (HI) $[5,8-13]$. As more research studies became available, the concept of CCSVI as a pathologic condition, its diagnostic utility and clinical impact for MS patients have been questioned, given that no causal relationship between CCSVI and MS has been confirmed [6, 14]. A recent meta-analysis, including available studies investigating the association of CCSVI frequency with MS, reported considerable heterogeneity across the studies and a conservative sensitivity analysis yielded null association [14].

Although there is abundant cross-sectional data on this subject [6], there were only a few studies examining the association of CCSVI and clinical outcomes in MS patients over short-term [7, 15-19], while no long-term prospective studies have been reported, thus far. Therefore, the objective of this study was to explore whether there is an association between presence and severity of variations in extracranial venous anatomy, indicative of
CCSVI, and clinical outcomes in patients with MS over 5 years.

\section{Methods \\ Subjects}

This 5-year follow-up is a part of an ongoing singlecenter, longitudinal, observational, case-control Cardiovascular, Environmental and Genetic risk factor in MS (CEG-MS) study that is recruiting subjects in Buffalo, NY [20]. The study was approved by the local Institutional Review Board of the University of Buffalo, USA and University of Naples, Italy, and written informed consent was obtained from each participant. The inclusion criteria for this follow-up CEG sub-study of variations in extracranial venous anatomy and clinical outcomes are: a) subjects originally enrolled in the CEGMS study in years 2009 through 2013 [5, 20], b) age 1875 years at baseline, and c) being MS patients or HI with unknown history of neurological disease. Exclusion criteria are: a) presence of relapse and steroid treatment within 30 days preceding their enrollment in the study (for MS patients), b) pre-existing medical conditions known to be associated with cerebral pathology (cerebrovascular disease, positive history of alcohol abuse), c) evidence of brain ischemic or hemorragic infarcts, or space-occupying lesions on MRI exam performed within 30 days of physical/neurologic examination with the standardized study protocol, and d) pregnancy. In total, 128 consecutive subjects consisting of 90 MS patients (52 with relapsing-remitting, RR and 38 with secondaryprogressive, SP disease subtype) and 38 HI with unknown history of neurological disease were enrolled in this CEG-MS sub-study by November 2016, when the first database lock occurred. Seven MS patients enrolled in the study had undergone percutaneous venous angioplasty at external centers for the treatment of their CCSVI at independent time points unrelated to the current study.

\section{Clinical examination}

MS was defined using the revised McDonald criteria [21] and all subjects were examined with physical and neurological examination by experienced neurologists, who were blinded to the results of the DS examination. Expanded Disability Severity Scale (EDSS) score was obtained for each MS patient at baseline and at follow-up. An absolute change in EDSS ( $\triangle E D S S)$ was calculated, and development of disability progression (DP) was documented as a clinical measure of irreversible neurological disability. DP was defined as an absolute change in EDSS over 5 years with an increase in EDSS of $\geq 1.5$ point if the baseline EDSS was 0 , or $\geq 1.0$ point if the baseline was between $1.0-5.5$, or $\geq 0.5$ point if the baseline EDSS was $\geq 6.0$. The medical history and relapses 
were documented during the 5-year follow-up study assessment.

\section{Doppler sonography assessment}

Doppler assessment was performed at baseline and at follow-up on all study participants using the same echo color Doppler machine, MyLab 25 Gold (Esaote-Biosound, Genoa, Italy) equipped with $2.5 \mathrm{MHz}$ and $7.5-10$ $\mathrm{MHz}$ transducers, and by a single Doppler sonographer who was blinded to the disease status of the subjects. The baseline blinding was achieved, by instructing subjects not to reveal their disease status during the examination, including patients with no disability or walking difficulties to ensure blinding between non-disabled MS patients and HIs [5]. At the follow-up, the blinding was achieved by positioning subjects supine in a tilt chair by a non-blinded personnel, draping from neck down and removing any assistive device used by the patients from the room before the Doppler sonographer entered, to avoid any visual cues of the presence of disease. The reproducibility of our approach was reported previously [5].

Detailed description of the baseline DS assessment of the extracranial (IJV and vertebral veins, VV) and intracranial venous system was previously reported [5]. Briefly, each subject was examined on a tilt chair in supine $\left(0^{\circ}\right)$ and sitting position $\left(90^{\circ}\right)$ allowing a minimum of 2 min post positional change for vascular flow redistribution prior to DS assessment. At the follow-up, the subjects were also examined on a tilt chair in supine and sitting position. All baseline and follow-up exams were examined in a blinded manner by a certified centralized reader (MM) at the University of Naples, Italy. The detection of extracranial venous variations in extracranial venous anatomy focused on the detection of 5 anomalous venous hemodynamic $(\mathrm{VH})$ criteria [5], with presence of $\geq 2 \mathrm{VH}$ criteria indicating the fulfillment of CCSVI criteria, according to the International Society for Neurovascular Disease (ISNVD) consensus statement [22]. Due to the technical discrepancy in the assessment of the $\mathrm{VH}$ criteria at baseline and at the follow-up, the $\mathrm{VH}$ criteria 1 and 2 were not comparable between the two time-points [22].

\section{Statistical analyses}

Statistical analysis was performed using the IBM Statistical Package for the Social Sciences (SPSS version 24.0). Student t-test, chi-square test and Mann Whitney U-test were used testing the differences between study groups, as appropriate. Wilcoxon signed-rank test was used to compare the individual $\mathrm{VH}$ criteria within the study groups between the baseline and the follow-up. As there was no significant difference observed in age, gender or disease duration between the study groups (MS vs HIs), chi-square test or Wilcoxon signed-rank test was used to investigate the association between the CCSVI status or the individual venous hemodynamic criteria. The evolution of clinical outcome measures (annualized relapse rate, ARR, $\triangle E D S S$ and DP) were analyzed using independent-sample t-test, Mann Whitney U-test and chi-square test as appropriate. A nominal $p$-value of $\leq 0.05$ was considered statistically significant, and $p<0.1$ was considered a trend, using two-tailed test.

\section{Results \\ Demographic and clinical characteristics}

The demographics and clinical characteristics of the study subjects are presented in Table 1 . The mean age at baseline was 45.1 years in HIs and 47.3 years in MS patients $(p=0.387)$. The average follow-up duration was similar in MS patients and HIs $(p=0.342)$. No significant differences were observed in the confounding variables between the study groups in terms of their gender, body mass index (BMI), prevalence of pre-existing comorbidities such as hypertension, hyperlipidemia or diabetes mellitus.

In the MS group, 7 (8\%) of the patients had progressed from clinically isolated syndrome (CIS) to relapsingremitting (RR) MS disease subtype, and 10 (11\%) from RRMS to secondary-progressive (SP) MS (Table 1). The DP was observed in $25(28 \%)$ of the patient population (Table 1). Eighty (89\%) of MS patients were on diseasemodifying treatment at the time of their follow-up visit (Table 1). Median $\triangle$ EDSS over the follow-up was 0.5, mean annualized ARR was 0.2 per year, and $61 \%$ of the patients remained relapse-free from baseline to follow-up.

Detailed demographic and clinical characteristics of the RRMS and SPMS patients at baseline are provided in the Additional file 1: Table S1. Demographic data was also compared between subjects fulfilling and notfulfilling ISNVD CCSVI criteria at baseline, to understand the influence of these variables on the outcome measures over the follow-up (Table 2). No significant differences were observed between these groups in terms of age, disease duration, BMI, comorbidities or followup duration.

\section{Venous hemodynamic differences between and within study groups}

Table 3 shows individual $\mathrm{VH}$ criteria and frequency of subjects fulfilling ISNVD CCSVI criteria in HIs and MS patients at baseline, and at the follow-up. At baseline, 52 (58\%) of MS patients and 14 (37\%) of HIs $(p=0.03)$ fulfilled ISNVD CCSVI criteria. At the follow-up, the ISNVD CCSVI criteria were fulfilled in $55(61 \%)$ of MS patients and $21(56 \%)$ of HIs $(p=0.486)$.

At the baseline visit, there was a significantly higher prevalence of positive $\mathrm{VH}$ criterion 3 in MS patients compared to HIs (54 vs $14, p=0.011$ ). At the 5 -year 
Table 1 Demographic and clinical characteristics of the study participants

\begin{tabular}{|c|c|c|c|}
\hline & $\mathrm{HI}(n=38)$ & MS $(n=90)$ & $p$ value \\
\hline Female, n (\%) & $25(67)$ & $66(73)$ & 0.387 \\
\hline Baseline age (yrs); mean (SD) & $45.1(13.6)$ & $47.3(10.4)$ & 0.309 \\
\hline Time to F/up (yrs); mean (SD) & $5.4(0.3)$ & $5.5(0.5)$ & 0.342 \\
\hline Baseline BMI $\left(\mathrm{kg} / \mathrm{m}^{2}\right)$; mean (SD) & $26.6(5.8)$ & $27.7(5.9)$ & 0.363 \\
\hline Follow-up BMI $\left(\mathrm{kg} / \mathrm{m}^{2}\right)$; mean (SD) & $26.2(5.2)$ & $27.9(5.9)$ & 0.363 \\
\hline \multicolumn{4}{|l|}{ Baseline comorbidities, n (\%) } \\
\hline Hypertension & $6(16)$ & $11(12)$ & 0.586 \\
\hline Hyperlipidemia & $6(16)$ & $11(12)$ & 0.586 \\
\hline Diabetes & $2(5)$ & $2(4)$ & 0.372 \\
\hline \multicolumn{4}{|l|}{ Follow-up comorbidities, n (\%) } \\
\hline Hypertension & $9(24)$ & $15(17)$ & 0.353 \\
\hline Hyperlipidemia & $10(26)$ & $22(23)$ & 0.719 \\
\hline Diabetes & $3(8)$ & $4(4)$ & 0.433 \\
\hline Baseline disease duration (yrs), mean (SD) & N/A & $15.3(10.0)$ & N/A \\
\hline \multicolumn{4}{|l|}{ Disease progression, n (\%) } \\
\hline $\mathrm{ClS} \rightarrow \mathrm{RR}$ & N/A & $7(8)$ & N/A \\
\hline $\mathrm{RR} \rightarrow \mathrm{SP}$ & & $11(13)$ & \\
\hline Remained RR & & $46(51)$ & \\
\hline SP since baseline & & $27(30)$ & \\
\hline Baseline EDSS, median (IQR) & N/A & $3.0(1.5-5.5)$ & N/A \\
\hline F/up EDSS, median (IQR) & N/A & $3.5(2.0-6.0)$ & N/A \\
\hline$\triangle \mathrm{EDSS}$, mean (SD); median & N/A & $0.3(0.9) ; 0.5$ & N/A \\
\hline DP at F/up, n (\%) & N/A & $25(28)$ & N/A \\
\hline Annualized relapse rate at F/up, mean (SD) & N/A & $0.2(0.4)$ & N/A \\
\hline Relapse free from baseline to follow-up, n (\%) & N/A & $55(61)$ & N/A \\
\hline \multicolumn{4}{|l|}{ Baseline DMT status, n (\%) } \\
\hline Interferon-beta 1a & $\mathrm{N} / \mathrm{A}$ & $34(38)$ & N/A \\
\hline Glatiramer acetate & & $20(22)$ & \\
\hline Natalizumab & & $18(20)$ & \\
\hline Other DMT* & & $4(4)$ & \\
\hline No DMT & & $14(16)$ & \\
\hline \multicolumn{4}{|l|}{ Follow-up DMT status, n (\%) } \\
\hline Remained on same DMT & N/A & $47(52)$ & N/A \\
\hline Switched to another DMT & & $33(37)$ & \\
\hline No DMT & & $10(11)$ & \\
\hline
\end{tabular}

HI Healthy individual, MS multiple sclerosis, $n$ number, SD standard deviation, BMI Body Mass Index, yrs Years, F/up Follow-up, CIS Clinically isolated syndrome, RR relapsing-remitting, $S P$ secondary-progressive, DP disability progression, EDSS Expanded Disability Status Scale, $\triangle E D S S$ Absolute change in EDSS, IQR interquartile range, DMT disease-modifying treatment

All p-values were calculated using independent-sample t-test, Mann Whitney U-test and chi-square test as appropriate

*Other DMTs include intravenous immunoglobulin, mitoxantrone and azathioprine

follow-up visit, there was a significantly higher prevalence of positive $\mathrm{VH}$ criterion 5 in MS patients compared to HIs (13 vs $0, p=0.018$ ), as none of the HIs presented with negative $\triangle$ CSA.

Wilcoxon signed-rank test showed no within group changes in VH criteria positivity or the fulfillment of the
ISNVD CCSVI criteria in MS patients and HIs between baseline and the follow-up. A trend toward significance was observed in $\mathrm{VH}$ criterion 5 positivity in MS patients from baseline to follow-up ( $\mathrm{Z}=-1.732 ; p=0.083)$. On further stratification of MS patients, 9 (24\%) of SPMS had positive $\mathrm{VH}$ criterion 5 at the follow-up, which was 
Table 2 Demographic and clinical characteristics of the multiple sclerosis patients, according to their fulfillment of CCSVI criteria at baseline

\begin{tabular}{|c|c|c|c|}
\hline & CCSVI ISNVD criteria fulfilled [22] $(n=52)$ & CCSVI ISNVD criteria not-fulfilled [22] $(n=38)$ & $p$ value \\
\hline Female, n (\%) & $37(71)$ & $29(76)$ & 0.584 \\
\hline Baseline age (yrs); mean (SD) & $47.6(10.7)$ & $47(10.0)$ & 0.815 \\
\hline Time to F/up (yrs); mean (SD) & $5.5(0.5)$ & $5.5(0.4)$ & 0.810 \\
\hline Baseline BMI $\left(\mathrm{kg} / \mathrm{m}^{2}\right)$; mean (SD) & $27.3(5.4)$ & $28.7(6.5)$ & 0.996 \\
\hline Follow-up BMI $\left(\mathrm{kg} / \mathrm{m}^{2}\right)$; mean (SD) & $29.1(4.9)$ & $27.2(6.5)$ & 0.212 \\
\hline \multicolumn{4}{|l|}{ Baseline comorbidities, n (\%) } \\
\hline Hypertension & $4(8)$ & $7(18)$ & 0.125 \\
\hline Hyperlipidemia & $6(12)$ & $5(13)$ & 0.817 \\
\hline Diabetes & $1(2)$ & $1(3)$ & 0.822 \\
\hline \multicolumn{4}{|l|}{ Follow-up comorbidities, n (\%) } \\
\hline Hypertension & $7(13)$ & $8(22)$ & 0.075 \\
\hline Hyperlipidemia & $13(24)$ & $8(22)$ & 0.644 \\
\hline Diabetes & $2(4)$ & $2(6)$ & 0.652 \\
\hline Baseline disease duration (yr), mean (SD) & $15.0(10.2)$ & $14.4(9.5)$ & 0.747 \\
\hline \multicolumn{4}{|l|}{ Disease progression, $\mathrm{n}(\%)$} \\
\hline $\mathrm{ClS} \rightarrow \mathrm{RR}$ & $3(6)$ & $4(11)$ & N/A \\
\hline $\mathrm{RR} \rightarrow \mathrm{SP}$ & $7(14)$ & $4(11)$ & \\
\hline Remained RR & $25(48)$ & $21(55)$ & \\
\hline SP since baseline & $17(33)$ & $9(24)$ & \\
\hline \multicolumn{4}{|l|}{ Baseline DMT status, n (\%) } \\
\hline Interferon-beta 1a & $17(33)$ & $17(48)$ & N/A \\
\hline Glatiramer acetate & $13(25)$ & $7(18)$ & \\
\hline Natalizumab & $13(25)$ & $5(13)$ & \\
\hline Other DMT* & $1(2)$ & $2(5)$ & \\
\hline No DMT & $8(15)$ & $6(16)$ & \\
\hline \multicolumn{4}{|l|}{ Follow-up DMT status, n (\%) } \\
\hline Remained on same DMT & $27(52)$ & $22(58)$ & N/A \\
\hline Switched to another DMT & $17(33)$ & $13(34)$ & \\
\hline No DMT & $8(15)$ & $3(8)$ & \\
\hline
\end{tabular}

$n$ number, SD standard deviation, BMI Body Mass Index, yr Years, F/up Follow-up, CIS Clinically isolated syndrome, RR relapsing-remitting, SP secondaryprogressive, DMT disease-modifying treatment ISNVD-International Society for Neurovascular disease

All $p$-values were calculated using independent-sample t-test, Mann Whitney U-test and chi-square test as appropriate

*Other DMTs include intravenous immunoglobulin, mitoxantrone and azathioprine

significantly greater compared to baseline $(Z=-2.33$; $p=0.02)$.

\section{Evolution of clinical outcomes according to the baseline fulfillment of the ISNVD CCSVI criteria}

Table 4 shows evolution of clinical outcomes, according to the baseline fulfillment of the ISNVD CCSVI criteria. At baseline, there was a trend for higher EDSS in those who fulfilled, compared to those who did not fulfill the criteria $(p=0.08)$, which was not evident at the followup $(p=0.11)$. No differences between those who fulfilled ISNVD CCSVI criteria and those who did not were observed over the follow-up in $\triangle \mathrm{EDSS}$, development of DP, number of the relapses between baseline and follow-up, ARR or being relapse-free. No differences in evolution of clinical outcomes was found, according to the baseline fulfillment of the ISNVD CCSVI criteria when RRMS and SPMS patients were considered separately.

\section{Clinical outcomes of MS patients who underwent} percutaneous venous angioplasty

Of 7 MS patients who underwent percutaneous angioplasty, 3 (42.9\%) were in RRMS and 4 (57.1\%) in the SPMS group. Six (86\%) of those fulfilled ISNVD CCSVI criteria at baseline and their median EDSS was 4.0. Median time period from the baseline to the date of procedure was 3 
Table 3 Comparison of venous hemodynamic Doppler sonography criteria at baseline and at follow-up study visit in healthy individuals and multiple sclerosis patients

\begin{tabular}{|c|c|c|c|}
\hline VH Criteria at Baseline & $\mathrm{HI}(\mathrm{n}=38)$ & MS $(n=90)$ & $p$-value \\
\hline Criterion 1 positivity, n (\%) - IJV and/or W reflux & $8(21)$ & $32(36)$ & 0.09 \\
\hline Criterion 2 positivity, n (\%) - Bidirectional IC venous outflow & $10(26)$ & $29(32)$ & 0.459 \\
\hline Criterion 3 positivity, $\mathrm{n}(\%)$ - B- mode IJV stenosis & $14(37)$ & $54(60)$ & 0.011 \\
\hline Criterion 4 positivity, $\mathrm{n}(\%)$ - No flow in IJV and/or WV & $3(8)$ & $10(11)$ & 0.557 \\
\hline Criterion 5 positivity, $\mathrm{n}(\%)-\operatorname{IJV} \operatorname{CSA} 90^{\circ}>\operatorname{IJV} \mathrm{CSAO}{ }^{\circ}$ & $1(3)$ & $6(7)$ & 0.346 \\
\hline CCSVI criteria fulfilled according to the ISNVD consensus [22], n (\%) & $14(37)$ & $52(58)$ & 0.03 \\
\hline VH Criteria at the follow-up & $\mathrm{HI}(\mathrm{n}=38)$ & MS $(n=90)$ & $p$-value \\
\hline Criterion 1 positivity, n (\%) - IJV and/or W reflux & $0(0)$ & $3(3)$ & 0.255 \\
\hline Criterion 2 positivity, n (\%) - Bidirectional IC venous outflow & $29(32)$ & $77(86)$ & 0.206 \\
\hline Criterion 3 positivity, $\mathrm{n}(\%)$ - B- mode IJV stenosis & $26(68)$ & $55(61)$ & 0.433 \\
\hline Criterion 4 positivity, $\mathrm{n}(\%)$ - No flow in IJV and/or WV & $7(18)$ & $17(19)$ & 0.951 \\
\hline Criterion 5 positivity, n (\%) - IJV CSA90 >IJV CSAO & $0(0)$ & $12(13)$ & 0.018 \\
\hline CCSVI criteria fulfilled according to the ISNVD consensus [22], n (\%) & $21(56)$ & $55(61)$ & 0.486 \\
\hline
\end{tabular}

CCSVI Chronic cerebrospinal venous insufficiency, $n$ number, $V H$ Venous hemodynamic, $H I$ Healthy individual, MS Multiple sclerosis, IJV Internal jugular vein, $V V$ Vertebral vein, IC intra-cranial, CSA Cross sectional area, ISNVD International Society for Neurovascular Disease

All $p$-values were calculated using Chi square and Fisher's exact test, and $p$ values $<0.05$ were considered significantt

months. At their follow-up, $57 \%$ patients continued to have fulfilled ISNVD CCSVI criteria. The mean $\triangle$ EDSS was 0.2 in RRMS and 0.8 in SPMS patients, respectively over the follow-up. DP was detected in 2 of the 7 patients (33\%) and the mean ARR of this subgroup was 0.13.

\section{Discussion}

To the best of our knowledge, this is the longest followup study assessing the longitudinal relationship between the presence of variations in extracranial venous anatomy and clinical outcomes in MS patients. The association of variations in extracranial venous anatomy with disability status in MS patients has been a debatable topic over the last decade. Our study found that the presence of variations in extracranial venous anatomy is not exclusive to the MS patients, as there was no significant difference in the prevalence of those in MS patients versus HIs, at the follow-up. Thus, a causal association between the two cannot be implied. Furthermore, a very small subset of our study population underwent venous angioplasty which did not alter the disease course in these patients. Due to the small sample size, these results are only descriptive and no statistical tests have been performed on these data.

The heterogeneity of the extracranial and intracranial venous system was documented using various imaging modalities including DS, MR or contrast venography [6]. The capacitance of the extracranial neck vessels is highly sensitive to alteration in patient position, hydration status and use of imaging methodology, thus, making standardized assessment challenging, especially longitudinally and particularly using DS [6]. These practical limitations should be kept in mind, when interpreting results of this follow-up study.

With total of $20 \%$ of the patients progressing from CIS to RR or from RR to SP MS, and DP occurring in $28 \%$

Table 4 Evolution of clinical outcomes over 5 years in multiple sclerosis patients, according to their baseline CCSVI status

\begin{tabular}{|c|c|c|c|c|}
\hline & Total $(n=90)$ & $\begin{array}{l}\text { CCSVI CCSVI ISNVD criteria } \\
\text { fulfilled [22] }(n=52)\end{array}$ & $\begin{array}{l}\text { CCSVI CCSVI ISNVD criteria } \\
\text { not-fulfilled [22] }(n=38)\end{array}$ & $p$ value \\
\hline EDSS at baseline, median (IQR) & $3.0(1.5-5.5)$ & $3.5(2.0-6.0)$ & $2.5(1.5-4.0)$ & 0.08 \\
\hline EDSS at follow-up, median (IQR) & $3.5(2.0-6.0)$ & $3.5(1.7-6.5)$ & $3.0(2.0-4.0)$ & 0.11 \\
\hline$\triangle E D S S$, mean (SD); median & $0.3(0.9) ; 0.5$ & $0.4(0.7) ; 0.5$ & $0.2(1.0) ; 0.3$ & 0.76 \\
\hline DP at follow-up, n (\%) & $25(28)$ & $16(31)$ & $9(24)$ & 0.50 \\
\hline Number of relapses between baseline and follow-up, mean (SD) & $0.9(2.0)$ & $0.8(1.6)$ & $1.0(2.5)$ & 0.52 \\
\hline Annual relapse rate over the follow-up, mean (SD) & $0.2(0.4)$ & $0.1(0.3)$ & $0.2(0.5)$ & 0.52 \\
\hline Relapse free from baseline to follow-up, $n(\%)$ & $55(61)$ & $33(64)$ & $22(58)$ & 0.60 \\
\hline
\end{tabular}

CCSVI Chronic cerebrospinal venous insufficiency, EDSS Expanded Disability Status Scale, $\triangle E D S S$ Absolute change in EDSS from baseline to follow-up, IQR interquartile range, $S D$ standard deviation, $n$ number, DP Disability progression, ISNVD International Society for Neurovascular Disease

All p-values were calculated using independent-sample t-test, Mann Whitney U-test and chi-square test as appropriate 
of the patients, this marks the first case-control study so far, to investigate the impact of variations in extracranial venous anatomy on disease progression in MS patients and its subtypes (relapsing vs progressive). At baseline, we found that prevalence of variations in extracranial venous anatomy was significantly higher in MS patients compared to HIs. However, no differences between MS patients and HIs was found at the follow-up.

The analysis of the current study has concluded on similar lines as our previously reported post hoc analysis of the cross-sectional CEG-MS study and other reports, which failed to document an association between CCSVI severity and clinical outcomes in MS patients [13, 23]. Although at baseline, there was a trend towards higher EDSS in patients fulfilling ISNVD CCSVI criteria, this pattern did not continue during our follow-up. In addition, we did not find apparent association between the baseline presence or severity of variations in extracranial venous anatomy and development of DP or change in $\triangle \mathrm{EDSS}$, indicating no predictive value in monitoring disability progression. This finding stresses the importance of conducting prospective studies when exploring associations between different conditions.

An interesting finding in our SPMS subgroup was that these patients demonstrated a significant lack of posturedependent IJV collapse (measured using negative IJV cross-sectional area, as part of the $\mathrm{VH}$ criterion 5), while a trend towards significance was observed similarly in the overall MS group. IJV collapse in orthostatism is a physiologic phenomenon influenced by decreased hydrostatic pressure [24]. To better understand the hemodynamic implications of the above, simultaneous dynamic assessment of cerebral venous outflow should be incorporated. Recent quantitative study of cerebral blood flow has implied an association between altered postural regulation of inflowoutflow in the cerebral vasculature of MS patients [25].

A concerning aspect of the hypothesis of etiopathogenic role of CCSVI in MS was the introduction of an interventional procedure to correct the deformity [26]. Use of percutaneous venous angioplasty has been complicated by serious adverse events such as fatal brainstem hemorrhage, iatrogenic cerebral venous thrombosis, stent migration and in-stent thrombosis, among others [27]. The PREMiSE, a double-blinded randomized trial for the use of venous angioplasty in MS patients demonstrated no benefit in clinical or radiological outcomes with use of venous angioplasty [7]. Another randomized, doubleblind, sham-controlled trial, Brave Dreams, has shown no clinical or radiological benefit of venous angioplasty for CCSVI in patients with MS [28]. Though, only $8 \%$ of our MS subset had undergone this procedure at outside facilities in the present study, no improvement in clinical outcomes was seen in our patients despite treatment for the underlying variations in extracranial venous anatomy.
The strengths of the present study include a prospective study design with a long follow-up duration of 5.5 years, the employment of blinding Doppler sonographer and neurologists to minimize the ascertainment bias between the study groups, as well as the use of blinded centralized reading of the baseline and follow-up Doppler examinations. All clinical outcome measures and Doppler parameters were assessed at the follow-up by the same personnel, as at baseline to avoid inter-rater variability.

However, a major limitation of this study was that there was a change in technical assessment of $\mathrm{VH}$ criteria 1 and 2 [22], which limited their use for comparison between the two studies timepoints. This further emphasizes that use of DS is not an adequate approach for monitoring variations in extracranial venous anatomy in both cross-sectional and longitudinal studies. Moreover, a number of recent multimodal diagnostic studies showed inadequate sensitivity and specificity of DS in screening real frequency of variations in extracranial venous anatomy $[7,8,29]$, which further outlines a need of applying multimodal approach to screen and monitor these variations in extracranial venous anatomy by using more accurate invasive and non-invasive imaging techniques $[8,22,30]$. Furthermore, Type II error may be a possibility due to limited sample size. However, we had an $80 \%$ power to detect a medium (0.60) effect (G*Power 3.1.9.2), suggesting the study was neither over- nor under-powered. Taken together with the highly similar 5 -year change in the clinical outcomes between groups $(p \geq 0.52)$, false negative are unlikely.

\section{Conclusions}

In conclusion, our findings showed that the presence of variations in extracranial venous anatomy was not associated with disease progression or increased clinical activity in MS patients over the 5-year follow-up.

\section{Additional file}

Additional file 1: Table S1. Demographics and clinical characteristics in multiple sclerosis patients, according to their disease subtype

\footnotetext{
Acknowledgements

The authors had full editorial control of the paper, and provided their final approval of all content.

\section{Author's contribution}

SG, KM, MM, BWG and RZ substantially contributed to the concept and design of the study. SG and JH performed the statistical analysis. SG and RZ drafted the first version of the article. SG, KM, MM, MGC, DJ, AC, JH, DH, CB, BWG and RZ were responsible for recruitment and accrual of data and revised the article critically for important intellectual content. All authors had access to the data. All authors read and approved the final manuscript
} 


\section{Funding}

This study was funded in part by the The Annette Funicello Research Fund for Neurological Diseases and internal resources of the Buffalo Neuroimaging Analysis Center. In addition, we received support from the Jacquemin Family Foundation.

Research reported in this publication was also funded in part by the National Center for Advancing Translational Sciences of the National Institutes of Health under award Number UL1TR001412. The content is solely the responsibility of the authors and does not necessarily represent the official views of the $\mathrm{NIH}$.

None of the funding agencies had any input in the design of the study; collection, analysis, and interpretation of data; and in writing the manuscript.

\section{Availability of data and materials}

Availability of data and materials will be made available on a reasonable request.

\section{Ethics approval and consent to participate}

The study was approved by the local Institutional Review Board of the University of Buffalo, USA and University of Naples, Italy and written informed consent was obtained by all study participants prior to enrollment. The IRB renewals were obtained annually on an ongoing basis throughout the study.

\section{Consent for publication}

Not applicable.

\section{Competing interests}

Sirin Gandhi, Karen Marr, Maria Grazia Caprio, Dejan Jakimovski, Avinash Chandra and Jesper Hagemeier have nothing to disclose.

Marcello Mancini received personal compensation from SDN S.p.A. David Hojnacki has received speaker honoraria and consultant fees from Biogen Idec, Teva Pharmaceutical Industries Ltd., EMD Serono, Pfizer Inc., and Novartis.

Channa Kolb has received speaker honoraria from Novartis, Genzyme and Biogen-Idec

Bianca Weinstock- Guttman received honoraria as a speaker and as a consultant for Biogen Idec, Teva Pharmaceuticals, EMD Serono, Genzyme\&Sanofi, Novartis and Acorda. Dr. Weinstock-Guttman received research funds from Biogen Idec, Teva Pharmaceuticals, EMD Serono, Genzyme\&Sanofi, Novartis, Acorda.

Robert Zivadinov received personal compensation from EMD Serono, Genzyme-Sanofi, Claret Medical, Celgene and Novartis for speaking and consultant fees. He received financial support for research activities from Roche-Genenetech, Teva Pharmaceuticals, Genzyme-Sanofi, Novartis, Claret Medical, Intekrin-Coherus and IMS Health.

\section{Author details}

${ }^{1}$ Buffalo Neuroimaging Analysis Center, Department of Neurology, Jacobs School of Medicine and Biomedical Sciences, University at Buffalo, State University of New York, Buffalo, NY, USA. ${ }^{2}$ Institute of Biostructure and Bioimaging, National Research Council of Italy, Rome, Italy. ${ }^{3}$ Jacobs Multiple Sclerosis Center, Department of Neurology, School of Medicine and Biomedical Sciences, University at Buffalo, State University of New York, Buffalo, NY, USA. ${ }^{4}$ Center for Biomedical Imaging at Clinical Translational Science Institute, University at Buffalo, State University of New York, Buffalo, NY, USA.

\section{Received: 1 October 2018 Accepted: 31 May 2019} Published online: 11 June 2019

\section{References}

1. Compston A, Coles A. Multiple sclerosis. Lancet. 2008;372:1502-17.

2. Rindfleisch E. Histologisches Detail zu der grauen Degeneration von Gehirn und Rückenmark. (Zugleich ein Beitrag zu der Lehre von der Entstehung und Verwandlung der Zelle.). Archiv für pathologische Anatomie und Physiologie und für klinische Medicin. 1863;26:474-83.

3. Adams CW. Perivascular iron deposition and other vascular damage in multiple sclerosis. J Neurol Neurosurg Psychiatry. 1988;51:260-5.
4. Zamboni P, Galeotti R, Menegatti E, Malagoni AM, Tacconi G, Dall'Ara S, et al. Chronic cerebrospinal venous insufficiency in patients with multiple sclerosis. J Neurol Neurosurg Psychiatry. 2009;80:392-9.

5. Zivadinov R, Marr K, Cutter G, Ramanathan M, Benedict RH, Kennedy C, et al. Prevalence, sensitivity, and specificity of chronic cerebrospinal venous insufficiency in MS. Neurology. 2011;77:138-44.

6. Zivadinov R, Chung CP. Potential involvement of the extracranial venous system in central nervous system disorders and aging. BMC Med. 2013;11:260

7. Siddiqui AH, Zivadinov R, Benedict RH, Karmon Y, Yu J, Hartney ML, et al. Prospective randomized trial of venous angioplasty in MS (PREMiSe). Neurology. 2014:83:441-9.

8. Traboulsee AL, Knox KB, Machan L, Zhao Y, Yee I, Rauscher A, et al. Prevalence of extracranial venous narrowing on catheter venography in people with multiple sclerosis, their siblings, and unrelated healthy controls: a blinded, case-control study. Lancet. 2014;383:138-45.

9. Baracchini C, Perini P, Calabrese M, Causin F, Rinaldi F, Gallo P. No evidence of chronic cerebrospinal venous insufficiency at multiple sclerosis onset. Ann Neurol. 2011;69:90-9.

10. Doepp F, Paul F, Valdueza JM, Schmierer K, Schreiber SJ. No cerebrocervical venous congestion in patients with multiple sclerosis. Ann Neurol. 2010;68: 173-83.

11. Tsivgoulis G, Mantatzis M, Bogiatzi C, Vadikolias K, Voumvourakis K, Prassopoulos $P$, et al. Extracranial venous hemodynamics in multiple sclerosis: a case-control study. Neurology. 2011;77:1241-5.

12. Patti F, Nicoletti A, Leone C, Messina S, D'Amico E, Lo Fermo S, et al. Multiple sclerosis and CCSVI: a population-based case control study. PLoS One. 2012;7:e41227.

13. Leone C, D'Amico E, Cilia S, Nicoletti A, Di Pino L, Patti F. Cognitive impairment and "invisible symptoms" are not associated with CCSVI in MS BMC Neurol. 2013;13:97.

14. Tsivgoulis G, Sergentanis TN, Chan A, Voumvourakis K, Triantafyllou N, Psaltopoulou T, et al. Chronic cerebrospinal venous insufficiency and multiple sclerosis: a comprehensive meta-analysis of case-control studies. Ther Adv Neurol Disord. 2014;7:114-36.

15. Denislic M, Milosevic Z, Zorc M, Ravnik IZ, Mendiz O. Disability caused by multiple sclerosis is associated with the number of extra cranial venous stenoses: possible improvement by venous angioplasty. Results of a prospective study. Phlebology. 2012.

16. Scalise F, Novelli E, Farina M, Barbato L, Spagnolo S. Venous hemodynamic insufficiency severity score variation after endovascular treatment of chronic cerebrospinal venous insufficiency. Phlebology. 2015;30:250-6.

17. Spagnolo S, Scalise F, Barbato L, Grasso MA, Tesler UF. Bilateral surgical reconstruction for internal jugular veins disease in patients with chronic cerebrospinal venous insufficiency and associated multiple sclerosis. Ann Vasc Surg. 2014;28:1793 e1791-4.

18. Zamboni P, Galeotti R, Menegatti E, Malagoni AM, Gianesini S, Bartolomei I, et al. A prospective open-label study of endovascular treatment of chronic cerebrospinal venous insufficiency. J Vasc Surg. 2009;50:1348-1358 e1341-1343.

19. Radak D, Kolar J, Sagic D, Ilijevski N, Tanaskovic S, Aleksic N, et al. Percutaneous angioplasty of internal jugular and azygous veins in patients with chronic cerebrospinal venous insufficiency and multiple sclerosis: early and mid-term results. Phlebology. 2014;29:367-75.

20. Zivadinov R, Ramasamy DP, Benedict RR, Polak P, Hagemeier J, Magnano C, et al. Cerebral microbleeds in multiple sclerosis evaluated on susceptibilityweighted images and quantitative susceptibility maps: a case-control study. Radiology. 2016;281:884-95.

21. Polman CH, Reingold SC, Banwell B, Clanet M, Cohen JA, Filippi M, et al. Diagnostic criteria for multiple sclerosis: 2010 revisions to the McDonald criteria. Ann Neurol. 2011;69:292-302.

22. Zivadinov R, Bastianello S, Dake MD, Ferral H, Haacke EM, Haskal Zl, et al. Recommendations for multimodal noninvasive and invasive screening for detection of extracranial venous abnormalities indicative of chronic cerebrospinal venous insufficiency: a position statement of the international society for neurovascular disease. J Vasc Interv Radiol. 2014;25:1785-1794 e1717.

23. Weinstock-Guttman B, Ramanathan M, Marr K, Hojnacki D, Benedict RH, Morgan C, et al. Clinical correlates of chronic cerebrospinal venous insufficiency in multiple sclerosis. BMC Neurol. 2012;12:26.

24. Valdueza JM, Doepp F, Schreiber SJ, van Oosten BW, Schmierer K, Paul F, et al. What went wrong? The flawed concept of cerebrospinal venous insufficiency. J Cereb Blood Flow Metab. 2013;33:657-68. 
25. Monti L, Menci E, Piu P, Leonini S, Arrigucci U, Bellini M, et al. A sonographic quantitative cutoff value of cerebral venous outflow in neurologic diseases: a blinded study of 115 subjects. AJNR Am J Neuroradiol. 2014;35:1381-6.

26. Ghezzi A, Annovazzi P, Cocco E, Coarelli G, Lugaresi A, Rovaris M, et al. Endovascular treatment of CCSVI in patients with multiple sclerosis: clinical outcome of 462 cases. Neurol Sci. 2013;34:1633-7.

27. Burton JM, Alikhani K, Goyal M, Costello F, White C, Patry D, et al. Complications in MS patients after CCSVI procedures abroad (Calgary, AB). Can J Neurol Sci. 2011;38:741-6.

28. Zamboni P, Tesio L, Galimberti S, Massacesi L, Salvi F, D'Alessandro R, et al. Efficacy and safety of extracranial vein angioplasty in multiple sclerosis: a randomized clinical trial. JAMA Neurol. 2018;75:35-43.

29. Zivadinov R, Karmon Y, Dolic K, Hagemeier J, Marr K, Valnarov V, et al. Multimodal noninvasive and invasive imaging of extracranial venous abnormalities indicative of CCSVI: results of the PREMiSe pilot study. BMC Neurol. 2013;13:151.

30. Dolic K, Siddiqui AH, Karmon Y, Marr K, Zivadinov R. The role of noninvasive and invasive diagnostic imaging techniques for detection of extra-cranial venous system anomalies and developmental variants. BMC Med. 2013;11:155.

\section{Publisher's Note}

Springer Nature remains neutral with regard to jurisdictional claims in published maps and institutional affiliations.

Ready to submit your research? Choose BMC and benefit from:

- fast, convenient online submission

- thorough peer review by experienced researchers in your field

- rapid publication on acceptance

- support for research data, including large and complex data types

- gold Open Access which fosters wider collaboration and increased citations

- maximum visibility for your research: over $100 \mathrm{M}$ website views per year

At BMC, research is always in progress.

Learn more biomedcentral.com/submissions 